\title{
Characterisation of the Surfactant Shell Stabilising Calcium Carbonate Dispersions in Overbased Detergent Additives: Molecular Modelling and Spin-Probe-ESR Studies
}

\author{
Francesco Frigerio and Luciano Montanari \\ ENI R\&M, SDM-CHIF, via Maritano 26, \\ 20097 San Donato Milanese, Italy \\ \{francesco.frigerio, luciano.montanari\}@eni.it
}

\begin{abstract}
The surfactant shell stabilising the calcium cabonate core in overbased detergent additives of lubricant base oils was characterised by computational and experimental methods, comprising classical force-field based molecular simulations and spin-probe Electron Spin Resonance spectroscopy. An atomistic model is proposed for the detergents micelle structure. The dynamical behaviour observed during diffusion simulations of three nitroxide spin-probe molecules into micelle models could be correlated to their mobility as determined from ESR spectra analysis. The molecular mobility was found to be dependent on the chemical nature of the surfactants in the micelle external shell.
\end{abstract}

\section{Introduction}

The lubrication of modern internal combustion engines requires the addition of specific additives to the base oils to improve the overall performance (minimization of corrosion, deposits and varnish formation in the engine hot areas) [1]. Calcium sulphonates are the most widely used metallic detergent additives. They are produced by sulfonation of synthetic alkylbenzenes. The simplest member would be a neutral alkylbenzene sulphonate with an alkyl solubilizing group approximately C18 to C20 or higher to provide adequate oil solubility. In addition to metallic detergents such as the neutral sulphonate, modern oil formulations contain basic compounds which provide some detergency. Their main function, however, is to neutralize acid and to prevent corrosion from acid attack. It is economically advantageous to incorporate as much neutralizing power in the sulphonate molecule as possible: excess base in the form of calcium carbonate can be dispersed in micelles $[2,3]$ to produce the so-called overbased sulphonates. Dispersions of calcium carbonate stabilized by calcium sulphonates have been characterized [4] using different techniques: electron microscopy [5], ultracentrifugation [6], and neutron scattering [7]. SAXS results show that overbased calcium sulphonates appear as polydisperse micelles having an average calcium carbonate core radius of $2.0 \mathrm{~nm}$ with a standard deviation of $0.4 \mathrm{~nm}$ [8]. The overbased calcium sulphonates form reverse micelles in oil, consisting of amorphous calcium 
carbonate nanoparticles surrounded by sulphonate surfactants. The polar heads (sulphonate) are attached to the metal core, while the hydrocarbon tails of hydrophobic nature stabilize the colloidal particle in the non-polar oil medium. Coupling three surface analyses techniques (XPS, XANES and ToF-SIMS) it was observed that there is a presence of some residual calcium hydroxide in the micellar core which is located prevalently at the surroundings of the micelle core [9]. ToF-SIMS shows that the molecular structures of detergent molecules are in good agreement with micelles synthesis data; little is still known on surfactant shell physical nature.

The compactness of the surfactant shells could play an important role to prevent negative consequences due to the interaction of carbonate core with other additives used in oil formulation or with water molecules. Such interactions cause the calcium carbonate separation by precipitation. In this study the molecular dynamics within the surfactant shell was probed in a combined computational and experimental approach by a small nitroxide such as TEMPO (2,2,6,6-tetramethylpiperidine-N-oxyl) and two nitroxide-labelled fatty acids (5- and 16-doxyl-stearic acids). In fact, nitroxides are known to exhibit ESR spectra that depend on the mobility of the spin-probe and the micro-viscosity of the probe environment. They have been used to evaluate the microstructure of the absorbed layer of surfactants and polymers at the solid-liquid interface [10-14] and also inside composite polymers [15, 16].

Detailed three-dimensional models were previously proposed for small overbased micelles containing various classes of surfactants [17-21]. Experimental measurements collected in our laboratory (data not shown) pointed to a couple of important micelle features: a flat disk shape for the inner core and a tightly packed outer shell. The core is mainly composed of amorphous calcium carbonate and it is surrounded by a distribution of surfactant molecules, arranged as a single layer with polar groups contacting the core surface. The diffusion of TEMPO and labelled fatty acids through the overbased micelle surfactant shell was simulated by a classical molecular mechanics methodology. The force-field based simulations protocols were applied to detailed atomistic models, which were built limited to the central portion of the micelle structure and contain all its essential features. The slow molecular motions of the stable surfactant layer around the rigid inorganic core could be reproduced by performing molecular dynamics calculations. Furthermore, the movements of the nitroxide spinprobes were studied by an approach combining forced diffusion and constraint-free molecular dynamics. Stable locations of such small molecules could be defined for each micelle model under investigation.

It is assumed that the polar head groups of the probe molecules (nitroxide for TEMPO and carboxylic for fatty acids) tend to be placed on the surface of the calcium carbonate cores. ESR spectra give information about the viscosity of the local environment at different distances from carbonate surface (at the boundary for TEMPO, while at 5- and 16- carbon positions for the two spin-labelled fatty acids). In our laboratory different surfactant molecules were used in the synthesis of overbased detergents with high calcium carbonate content, expressed as Total Base Number (the amount of soluble colloidal carbonate and of metal oxide and hydroxide, measured as equivalent milligrams of $\mathrm{KOH}$ per gram of sample [22]). Three overbased detergents with $\mathrm{TBN}=300$ and a mixture of mono- and di-alkyl-benzene-sulphonate were analyzed. 


\section{Experimental Methods}

An approach combining computer graphics and atomistic simulations [17, 19] produced a detailed model for the central portion of the overbased micelle structure. The essential model features (thickness and internal structure of the core, concentration and location of excess hydroxide ions, density and molecular arrangement of the shell) were inferred from analytical determinations performed on the detergent micelles that were produced in our laboratories (data not shown). Different relative concentrations of the surfactants were used to build the external shell of three micelle models, referred to as model a, b, c throughout this paper. The starting molecular and ionic building blocks were selected and manually manipulated within the InsightII [23] graphical interface in order to set up initial atomic distributions. A partial micelle model was built as an inorganic slab surrounded by two surfactant layers, one on top of each of its largest surfaces. Three-dimensional periodic boundary conditions were constantly applied during simulations in order to avoid model truncation effects. Atomic parameters were assigned from the pcff force field [24]. Afterwards an amorphous calcium carbonate core (with a small concentration of hydroxide ions) and a tight surfactant shell were generated by applying stepwise Monte Carlo docking and molecular dynamics [25] to limited portions of the micelle models. These simulations were performed by using InsightII and Discover [23], respectively. Along this part of the model building process, the uninvolved atoms were kept frozen. Nitroxide spinprobes were then added to the system assembly, after subjecting the obtained micelle models to extensive energy relaxation. The starting configurations contain a small cluster of spin-probe molecules, packed in a single layer contacting the micelle surfactant shell. Respectively, 14 molecules of TEMPO, 6 of 5-doxyl-stearic acid and 6 of 16-doxyl-stearic acid were used. The forced diffusion procedure available within InsightII [23] was carefully tailored to suit the overbased micelle model features. Its application followed the thorough energy minimisation of each one of the starting system configurations. Since the molecular motions within tightly packed assemblies are very slow, they were accelerated by adding a directional force for a very short time period at the beginning of the simulations, with the effect of gently pushing the nitroxide spin-probes toward the micelle core. In this way the extremely long process of generating spontaneous but unfrequent diffusion pathways could be avoided and the simulations were concentrated on the more interesting task of studying the small molecule motions throughout the micelle surfactant shell. The potential energy of the system and the relative distances between the nitroxide groups of the spin-probes and the micelle core center were derived by analysing the trajectories collected during the following free molecular dynamics simulations. These values were finally compared to the results from equivalent simulations, performed without the previous application of the forced diffusion protocol and used as a reference (non-diffusive) state.

All spin-probe molecules (Aldrich Chemie) were diluted at $0.3 \mathrm{mM}$ concentration into a mixture of SN150/SN500 (produced by ENI according to ASTM standard specification) lubricant bases (2/1 by weight). The overbased detergents were dissolved (at $30 \%$ by weight) into the spin-probe/lubricant solutions. The ESR spectra were collected with a Bruker ESP $300 \mathrm{E}$ spectrometer conditioned at a temperature of $50^{\circ} \mathrm{C}$. 


\section{Results}

The diffusion pathways of TEMPO and of 5- and 16- spin-probe labelled stearic acids were followed and compared in the three model-built partial micelles (identified as a, $b, c)$ with share an identical inorganic core but differ by the molecular distribution of surfactants in the external shell.
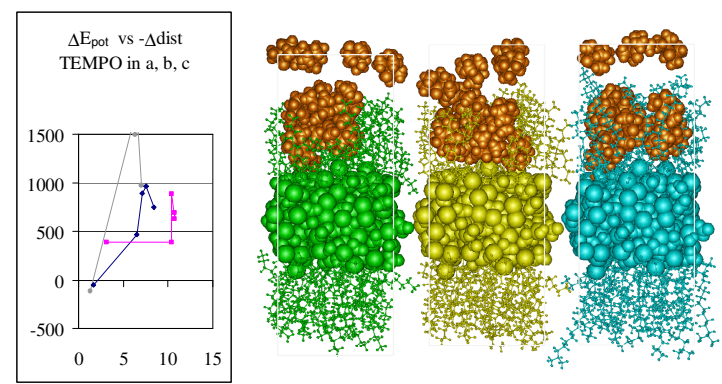

Fig. 1. Plot (left): $-\Delta$ distance $(\AA)$ vs $\Delta \mathrm{E}_{\text {potential }}\left(\mathrm{kcal} \cdot \mathrm{mol}^{-1}\right)$ from the simulation of TEMPO into micelle models a (grey), b (blue), c (magenta). Pictures (right): simulation boxes with 14 molecules (orange, Van der Waals) diffused into models a (green), b (yellow), c (cyan), composed of inorganic core (Van der Waals) and surfactant layers (ball and stick).

The force constant application produced diffusive pathways during dynamics trajectories (Fig. 1) for the micelle models containing TEMPO. The average penetration depth of the TEMPO nitroxide group into the micelle model is shortest and most energetically unfavourable (Fig. 1, left) within model a, while the results are slightly better with model $\mathrm{b}$ and at their best within model $\mathrm{c}$. The potential energy cost payed for the production of TEMPO diffusive pathways appears high and generally increasing with the force constant value. For fast comparison among the micelle models only very short trajectories were analysed. Further, longer molecular dynamics simulations (data not shown) completely release all strain accumulated during the first part of the
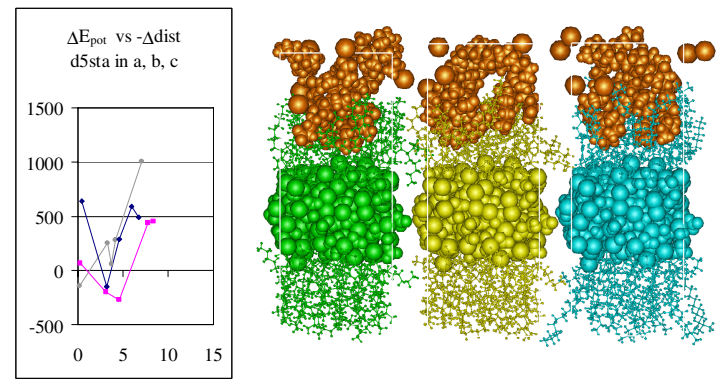

Fig. 2. Plot (left): $-\Delta$ distance $(\AA)$ vs $\Delta \mathrm{E}_{\text {potential }}\left(\mathrm{kcal} \cdot \mathrm{mol}^{-1}\right)$ from the simulation of 5-doxylstearic acid into micelle models a (grey), b (blue), c (magenta). Pictures (right): simulation boxes with 6 molecules (orange, Van der Waals) diffused into models a (green), b (yellow), c (cyan), composed of inorganic core (Van der Waals) and surfactant layers (ball and stick). 
diffusive process, while the final configurations do not differ significantly. One of the final, energetically relaxed TEMPO diffusion configurations is depicted for each of the three models a, b, c (Fig. 1, right). While most spin-probe molecules are located into the surfactant shell, only a few of them can get in contact with the carbonate core.

These results can be compared to the dynamic behaviour of 5-doxyl-stearic acid (Fig. 2). The average equilibrium penetration depth towards the micelle core (Fig. 2, left) is similar to what observed with TEMPO, but the generally lower potential energy cost reveals an easier diffusion through the surfactant shell. Anyway the structure of the two spin-probes is different: the stearic acid bears a nitroxide group laterally grafted to its long tail, therefore the reported distances from the micelle core (Fig. 2) apply to a longer molecule than in the case of TEMPO (Fig. 1). However, the order of spin-probe diffusion efficiencies through the three micelle models is again found as: $\mathrm{a}<\mathrm{b}<\mathrm{c}$. Three of the resulting relaxed configurations are reported (Fig. 2, right). Differently from TEMPO, only a small fraction of the labelled stearic acid molecules is able to reach a deep location into the surfactant shell.

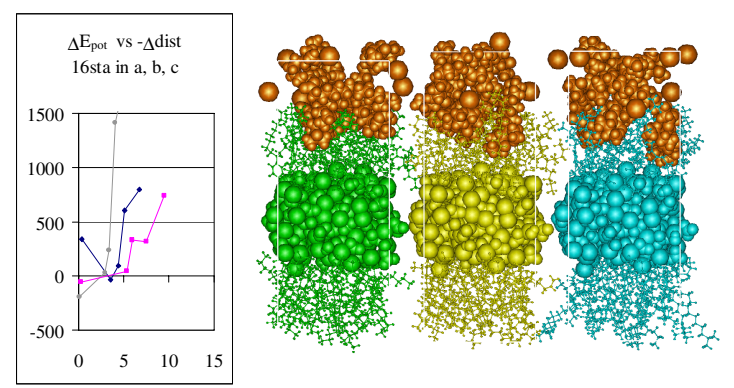

Fig. 3. Plot (left): $-\Delta$ distance $(\AA)$ vs $\Delta \mathrm{E}_{\text {potential }}\left(\mathrm{kcal} \cdot \mathrm{mol}^{-1}\right)$ from the simulation of 16-doxylstearic acid into micelle models a (grey), b (blue), c (magenta). Pictures (right): simulation boxes with 6 molecules (orange, Van der Waals) diffused into models a (green), b (yellow), c (cyan), composed of inorganic core (Van der Waals) and surfactant layers (ball and stick).

Comparable results were obtained from the analysis of the 16-doxyl-stearic acid dynamics trajectories (Fig. 3). The average equilibrium penetration depth (Fig. 3, left) plotted against the potential energy cost still reveals some differences: model $\mathrm{c}$ is slightly favoured over $\mathrm{b}$, and this last over a. Limited penetration into the surfactant shell is generally observed, compared to 5-doxyl-stearic acid. This can be attributed to the nitroxide group location further away from the the spin-probe polar head in the 16-doxyl-stearic acid. Three energetically relaxed configurations, resulting from the interaction of a cluster of 16-doxyl-stearic acid with models a, b, c, are reported (Fig. 3, right). As previously evidenced, the penetration of the surfactant shell by these spin-probe molecules is limited and they do not get in close contact with the core surface, differently from what happens with TEMPO.

The ESR spectra recorded for spin-probe molecules in solutions containing overbased micelles a, b and c are presented in Fig. 4, 5, and 6. Frequently such spectra show the superimposition of two components, an isotropic triplet produced by freely moving molecules and an anisotropic feature typical of a species located in a rigid 
environment. The characteristic shape of that anisotropic signal originates from the rotation of cylindrical molecules. In the adopted conditions $2 \mathrm{~A} / /$ hyperfine parallel coupling could be measured on most recorded spectra; its values are reported on Table 1 .

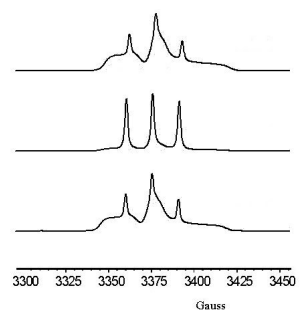

Fig. 4. ESR spectra of a solution of TEMPO in base lubricant oil containing, respectively, overbased micelles a (top), b (middle), c (bottom)

The results obtained with TEMPO (Fig. 4) clearly show the two components described above. The $2 \mathrm{~A} / /$ values (Table 1 ) for the three overbased micelles are quite similar to each other, though a somewhat lower rigidity is suggested for model a (Fig. 4, top). The aspect of ESR spectrum for model b (Fig. 4, middle) is dominated by the isotropic signal, revealing a lower population of the anisotropic species, compared to the other two overbased micelle solutions.

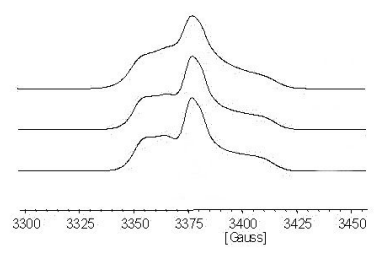

Fig. 5. ESR spectra of a solution of 5-doxyl-stearic acid in base lubricant oil containing, respectively, overbased micelles a (top), b (middle), c (bottom)

The 2A// values (Table 1) measured for 5-doxyl-stearic acid (Fig. 5) reveal a less rigid environment around the nitroxide group, as compared to TEMPO. This is slightly more evident for models b and c (Fig. 5, middle and bottom).

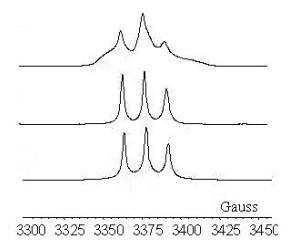

Fig. 6. ESR spectra of a solution of 16-doxyl-stearic acid in base lubricant oil containing, respectively, overbased micelles a (top), b (middle), c (bottom) 
With the 16-labelled stearic acid spin-probe (Fig. 6) a $2 \mathrm{~A} / /$ value could be measured (Table 1) only in the spectrum recorded for micelle a (Fig. 6, top), whereas in the other cases no hyperfine coupling could be detected by the peak analysis.

Table 1. Hyperfine parallel coupling 2A// values measured from the ESR spectra of overbased micelle solutions with three different spin-probes

\begin{tabular}{llll}
\hline Spin-Probe & TEMPO & 5-doxyl- stearic acid & 16-doxyl-stearic acid \\
\hline micelle a & 72.9 & 66.3 & 69 \\
micelle b & 74.5 & 64.5 & \\
micelle c & 74.1 & 63.5 & \\
\hline
\end{tabular}

The differences in mobility observed by comparing the ESR spin probe spectra mainly ensued from their diverse spin-label distances from the micelle core polar surface. The carboxyl group of both labelled fatty acids was strongly attracted but could not reach it through the surfactant shell. On the contrary TEMPO was able to penetrate deeply and produced the highest $2 \mathrm{~A} / /$ values. The larger coupling value for 5doxyl spin probe in micelle a is due to peculiar alkyl chain features of that surfactant shell. This effect is observed only with labelled fatty acids and is put into greater evidence when the nitroxide sits at a long distance from the micelle core as in 16-doxylstearic acid: the 2A// value is higher than for 5-doxyl-stearic acid in micelle a, while in micelles $\mathrm{b}$ and $\mathrm{c}$ the mobility is comparable to that of a free molecule in solution.

\section{Discussion}

The development of new generations of surfactants for lubricant oil additives requires an accurate characterisation of the reverse micelle structure of the overbased detergents [26]. In this study a combination of experimental and computational results helped defining a correlation between the chemical nature of the stabilising surfactant shell and the environmental rigidity imposed upon diffusing small molecules. The surfactant shell compactness, responsible for the remarkable stabilisation of the strongly basic micelle core in a non-polar environment, can be reasonably distinguished from shell viscosity. The first property is commonly attributed to a tight packing of surfactant aromatic moieties [19,21], while the second is mainly influenced by the molecular features of their alkyl chains. The ESR spectra analysis contributed a quantitative measurement of the micelle shell viscosity, revealing a subtle modulation of the mobility experienced by spin-probe molecules in different locations throughout the surfactant shell. The spin-probes diffusion was found to depend on both their molecular shape and the polar groups location along the structure. The molecular dynamics simulations of such process provided a pictorial description of the surfactant shell viscosity effects on diffusion. Moreover, its distance dependence from the micelle core was quantitatively confirmed. Small molecules like TEMPO were able to penetrate into a highly rigid environment next to the core surface, while labelled fatty acids were shown to fill the available room among surfactant alkyl chains, further away from the core. Compared with TEMPO, the nitroxide grafted next to the fatty acid polar group (5-doxyl-stearic acid) experienced higher mobility. When 
attached to the apolar end (16-doxyl-stearic acid) molecular freedom was found as high as in solution and an environmnetal rigidity effect was revealed only by the peculiar surfactant shell structure of micelle a. In conclusion, the previously defined structural features of overbased reverse micelles [2, 17-21, 26] have been further detailed by this study, in view of developing improved performances as detergent additives of lubricant base oils.

\section{References}

1. Liston, T. V., Lubr. Eng. 48 (1992) 389-397

2. Roman, J.P., Hoornaert, P., Faure, D., Biver, C., Jacquet, F., Martin, J.M., J. Coll. Interface Sci. 144 (1991) 324-339

3. Bandyopadhyaya, R., Kumar, R., Gandhi, K.S., Langmuir 17 (2001) 1015-1029

4. Hudson, L.K., Eastoe, J., Dowding, P.J., Adv. Coll. Interface Sci. 123-126 (2006) 425-431

5. Mansot, J.L. and Martin, J.B., J. Microsc. Spectrosc. Electron., 14 (1989) 78

6. Tricaud, C., Hipeaux, J.C., Lemerle, J., Lubr. Sci. Technol. 1 (1989) 207

7. Markovic, I., Ottewill, R.H., Coll. Polymer. Sci. 264 (1986) 454

8. Giasson, S., Espinat, D., Palermo, T., Ober, R., Pessah, M., Morizur, M.F., J. Coll. Interface Sci. 153 (1992) 355

9. Cizaire, L.; Martin, J.M.; Le Mogne, Th., Gresser, E., Coll. Surf. A 238 (2004) 151

10. Berliner, L.J.: Spin Labeling, Theory and Applications, Academic Press, New York (1979)

11. Dzikovski, B.G., Livshits, V.A., Phys. Chem. Chem. Phys. 5 (2003) 5271

12. Wines, T.H., Somasundaran, P., Turro, N.J., Jockusch, S., Ottaviani, M.F., J. Coll. Interface Sci. 285 (2005) 318

13. Kramer, G., Somasundaran, P., J. Coll. Interface Sci. 273 (2004) 115

14. Tedeschi, A.M., Franco, L., Ruzzi, M., Padano, L., Corvaja, C., D’Errico, G., Phys. Chem. Chem. Phys. 5 (2003) 4204

15. Maddinelli, G., Montanari, L., Ferrando, A., Maestrini, C., J. Appl. Polym. Sci. 102 (2006) 2810

16. Randy, B, Rabek, J.F.: ESR Spectroscopy in Polymer Research, Springer-Verlag, New York (1977)

17. Tobias, D.J., Klein, M.L., J. Phys. Chem. 100 (1996) 6637-6648

18. Griffiths, J.A., Bolton, R., Heyes, D.M., Clint, J.H., Taylor, S.E., J. Chem. Soc. Faraday Trans. 91 (1995) 687-696

19. Griffiths, J.A., Heyes, D.M., Langmuir, 12 (1996) 2418-2424

20. Bearchell, C.A., Danks, T.N., Heyes, D.M., Moreton, D.J., Taylor, S.E., Phys. Chem. Chem. Phys. 2 (2000) 5197-5207

21. Bearchell, C.A., Heyes, D.M., Moreton, D.J., Taylor, S.E., Phys. Chem. Chem. Phys. 3 (2001) 4774-4783

22. Arndt, E.R., Kreutz, K.L. J.Coll. Interface Sci. 123 (1988) 230

23. Accelrys, Inc., San Diego

24. Hill, J.-R., Sauer, J., J. Phys Chem. 98 (1994) 1238-1244

25. Allen, M.P., Tildesley, D.J.: Computer simulations of liquids, Clarendon, Oxford (1987)

26. Hudson, L.K., Eastoe, J., Dowding, P.J., Adv Colloid Interface Sci 123-126 (2006) 425431 